\title{
DEEP LEARNING-BASED MOTION ACTIVITY RECOGNITION USING SMARTPHONE SENSORS
}

\author{
Saedeh Abbaspour ${ }^{1,2}$, Faranak Fotouhi ${ }^{2}$, Hossein Fotouhi ${ }^{1}$, Maryam Vahabi ${ }^{1}$ and Maria Linden ${ }^{1}$ \\ ${ }^{1}$ School of Innovation, Design, and Engineering, Ma"lardalen University, Sweden \\ ${ }^{2}$ Faculty of Engineering, University of Qom, Qom, Iran
}

\begin{abstract}
MHealth systems establish a new way to transfer the health service to remote places. These systems offer significant benefits for continuous health monitoring. Motion activity recognition is one of the challenging mHealth use cases that incorporates continuous data collection and analysis of measurements. The main goal of this research is to analyze physical activity data. We employ measurements from the WISDM lab dataset ${ }^{1}$. These data are collected from participants performing motion activities. This data is then used by deep learning algorithms to predict special activities. In particular, CNN and CNN-LSTM algorithms are used to compare their accuracy, which resulted in approximately $95 \%$ and $97 \%$ respectively. Thus, the CNN-LSTM has higher accuracy in this analysis.
\end{abstract}

\section{KEYWORDS}

MHealth, Activity Recognition, Accelerometer, Deep Learning

\section{INTRODUCTION}

EHealth encompasses a wide variety of sub-domains of digital health such as telehealth and telemedicine, health IT system, electronic health record, and mobile health(mHealth). The recent advances in manufacturing mobile devices and the rise of wearable sensors embedded in people's everyday lives have led to the concept of mHealth monitoring. MHealth is defined as access and practice of healthcare via mobile telecommunication, multimedia technology, and wireless body sensors through a smartphone healthcare application (Adibi, 2015) (Choi, 2018). Figure 1 shows a schematic view of a typical mHealth system. In this system, the data collected from the sensor/actuator devices are sent to the cloud servers, where they are interpreted and analyzed. The analysis results are then submitted to both specialists in the clinic and the mobile application users. Any decision made by the specialists is then recorded in the servers and forwarded to the actuators.

The mobile applications can be categorized into two groups: (1) fitness, diet, and nutrition apps, and (2) disease and treatment management apps. According to Gartner, $40 \%$ of digital health systems in 2021 will shift to wearable devices which $90 \%$ will include fitness trackers (Lee, 2018). These mHealth apps could calculate the amount of energy consumed during physical activity. In fact, they use accelerometers, gyroscope (Fan, 2015), and GPS (Gusenbauer, 2010) (Jain, 2017)(Lee, 2018)(Sukaphat, 2013)(Rundle, 2016) to obtain and analyze the required data. For example, Hwang et al. have provided a fitness system embedded in shoes to measure steps, calorie, and biomedical information using the pressure sensors (Hwang, 2016).Adi et al. have built a system to measure the heart rate, ECG signal, step count, and calorie consumption using the Smartphone's accelerometer (Pande, 2015). Pande et al. used Smartphone sensors such as accelerometer and barometer to obtain energy expenditure (Kwapisz, 2011). Pal and Hepsiba estimated the user's energy expenditure using the Smartphone's accelerometer and GPS (Vathsangam, 2015). Duclos et al. have used accelerometer data to measure energy expenditure (Duclos, 2016). Li et al. used the ECG and accelerometer sensors to estimate energy expenditure ( $\mathrm{Li}, 2016)$. Altini et al. used five accelerometer sensors attached to the body to assess the energy expenditure during the daily activities (Altini, 2014). Carneiro et al. used a smartphone accelerometer to estimate energy expenditure (Carneiro, 2015). As a previous work, a wearable kit is designed to calculate calorie consumption. This system used a motion sensor and a PPG

1 The WISDM Lab dataset: http://www.cis.fordham.edu/wisdm/dataset.php 
(Photoplethysmography) sensor separately. The output of these sensors was sent to an android app by Bluetooth (Fotouhi-Ghazvini, 2020).

The majority of the above contributions rely on Smartphone embedded sensors to collect the physiological data, which hugely lacks precision and accuracy. For example, in (Asimina, 2018), the Fitbit was joint with a Smartphone app for step count, but it wrongly underestimated the step counts. In (Van Camp, 2017), the Fitbit was incorporated to measure the step counts though it was imprecise. Similarly, in the SmartWatch market for instance in (Schneider, 2016) and (Hamari, 2017), the ActiGraph was used which erroneously overestimated the frequency of the activity and the step counts. The Huawei and Apple watches include an accelerometer, gyroscope, heart rate, altimeter, and microphone sensors (King, 2018). In (Boletsis, 2015), the Basic B1 smartwatch was incorporated for dementia care at home. It provided the heart rate, the ambient temperature, and the activity levels. However, it did not reveal the exact activity during daily activities. In this paper, it is demonstrated how deep learning can help us to improve the accuracy of predictions. In fact, two well-known algorithms are elaborated and compared with respect to their accuracy. Thus, the main goal of this paper is to use deep learning algorithms to classify motion activities by using accelerometer data.

The rest of the paper is organized as follows. Section 2 discusses the input data used in the experiments. The deep learning algorithms used to analyze those data are explained in section 3 , and the analysis results are discussed in section 4. Finally, section 5 concludes the paper and outlines future directions.

\section{DATA COLLECTION}

In this paper, the data from WISDM dataset (Kwapisz, 2011) is used, which consists of cell phone accelerometer data. In particular, the values of acceleration are analyzed to detect the activity type by employing deep learning algorithms. The activities include "Walking", "Jogging", "Upstairs"," Downstairs", "Sitting" and "Standing". This dataset is extracted from twenty-nine participants. In the first step, the values of the accelerometer are used to predict activity type. These data can help us to monitor the motion activity for each participant. In this case, the participants carried the Android phone in their front pants leg pocket and were asked to walk, jog, ascend stairs, descend stairs, sit and stand for specific periods of time. The accelerometer data are collected every $50 \mathrm{~ms}$, so the sampling rate is $20 \mathrm{~Hz}$ per second.

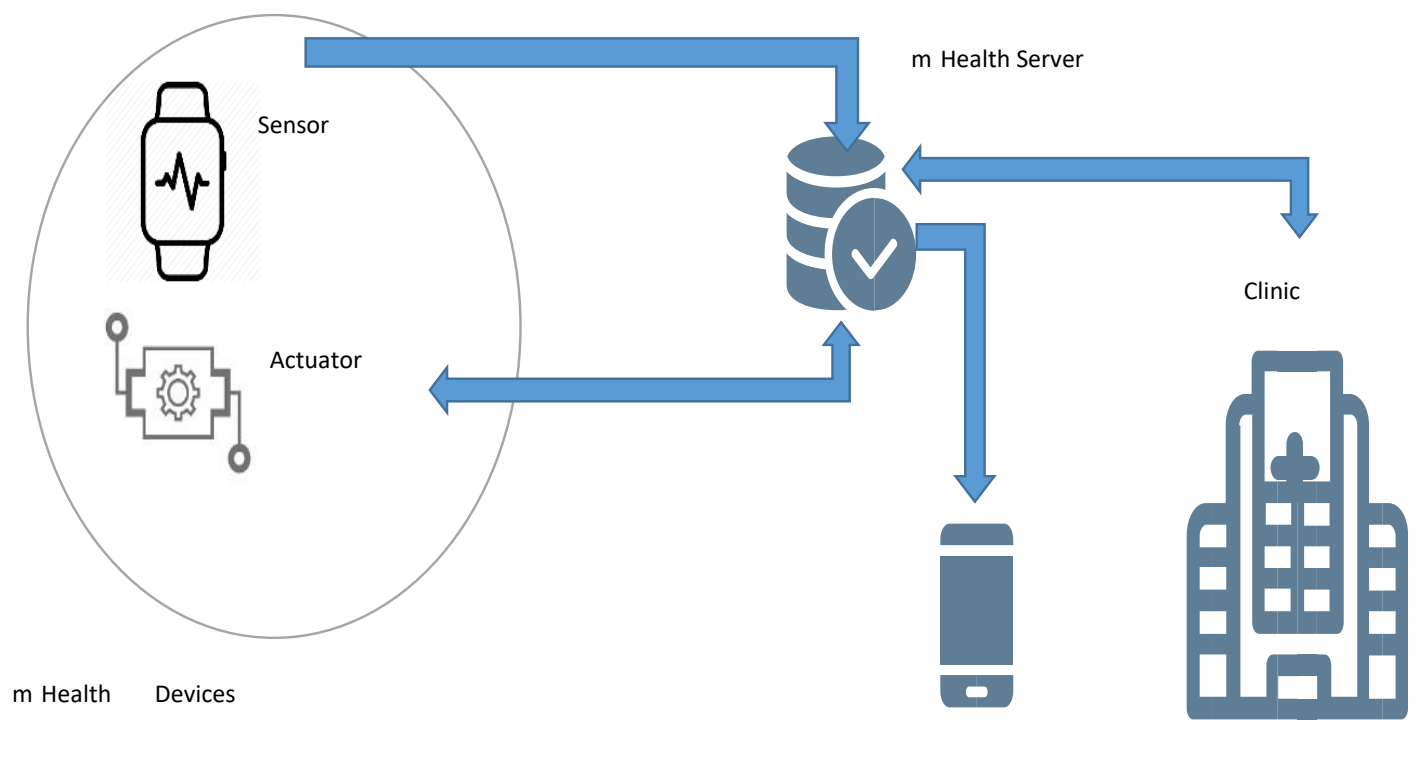

m Health Client

Figure 1. A schematic view of a typical mHealth system 


\section{DEEP LEARNING ALGORITHMS}

Deep learning is a subset of machine learning that relies on several layers of artificial neural networks (ANNs) to process unstructured data and detect patterns to be used in decision making. ANNs are inspired by the structure of the human brain and consist of layers, each representing a set of features. These features help the deep learning algorithm to extract the best model characterizing the input data.

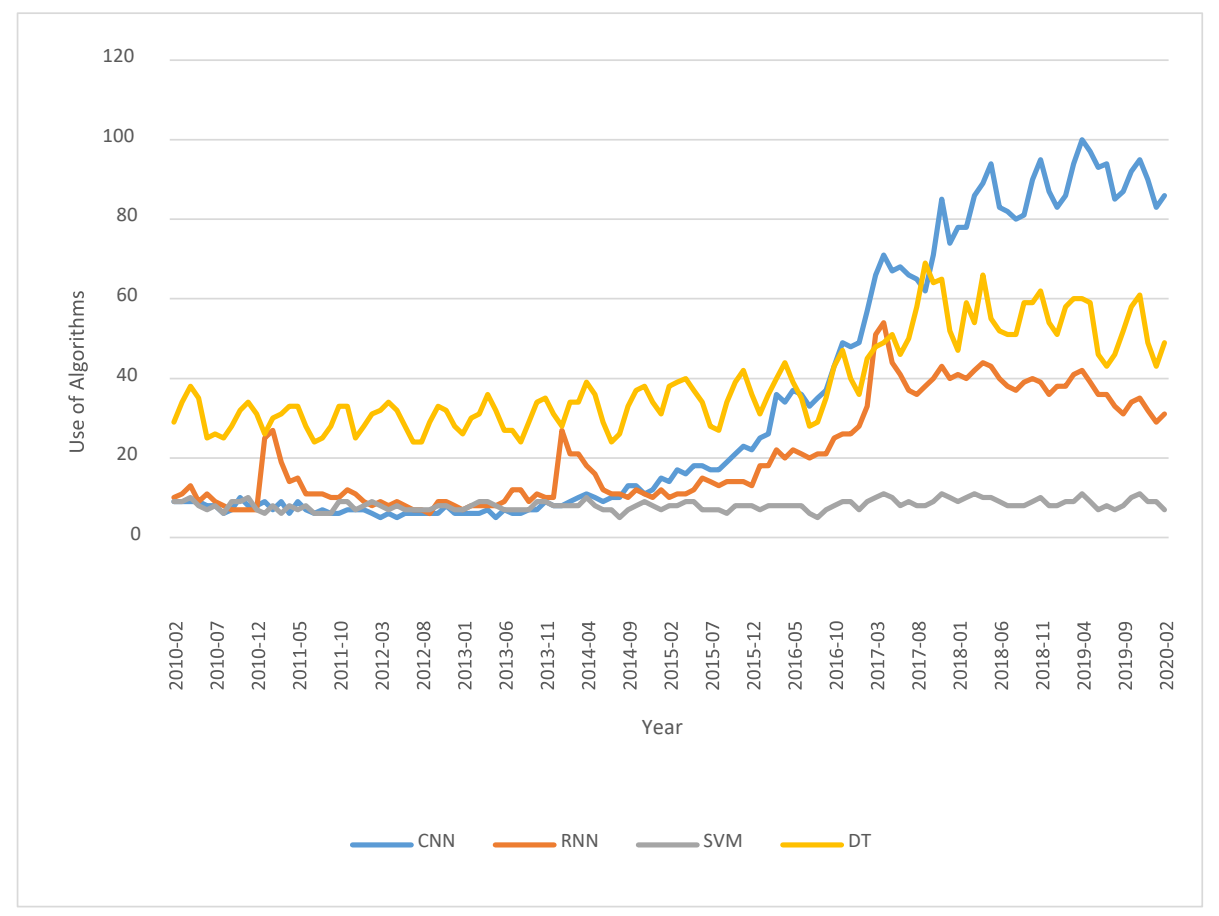

Figure 2. The use of deep learning and traditional algorithms

There are several types of deep algorithms each suitable for a different type of application. CNN (Convolutional Neural Network) and RNN (Recurrent Neural Network) are among the well-known deep algorithms. CNNs are feed forward ANNs widely used in computer vision for image recognition. However, RNNs include some feedback loops which make them more suitable for classifying time series data.

Deep learning can extract features automatically. Also, it works better than traditional machine learning algorithms. The greater the number of layers within the neural network, the better the features are extracted. And there is no need to calculate the feature manually. Traditional machine learning algorithms such as SVM (Support Vector Machine), KNN (K- Nearest Neighbors) and Decision Tree is dependent on human knowledge. However, deep learning algorithms ignore the presence of a human in the loop. Deep learning is an appropriate technique for obtaining accurate and fast classification. Figure 2 shows the use of deep learning and traditional algorithms in the last 10 years. Accordingly, the trend of using the CNN algorithm has increased enormously compared to other machine learning algorithms.

The main goal of this paper is to use deep learning algorithms to classify motion activities. These activities are extracted from WISDM dataset. In this case, acceleration values are used. These data are collected from smartphone sensor. The acceleration data are used as input of deep algorithms. Now, the feature extraction phase is performed automatically without expert supervision by using CNN and CNN-LSTM. As shown in figure 2 these two types of algorithms are more used. Figure 3 shows the structure of CNN and CNN-LSTM in our analysis. 


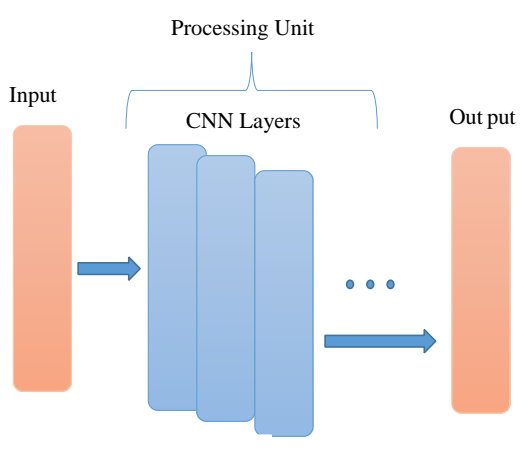

(a)

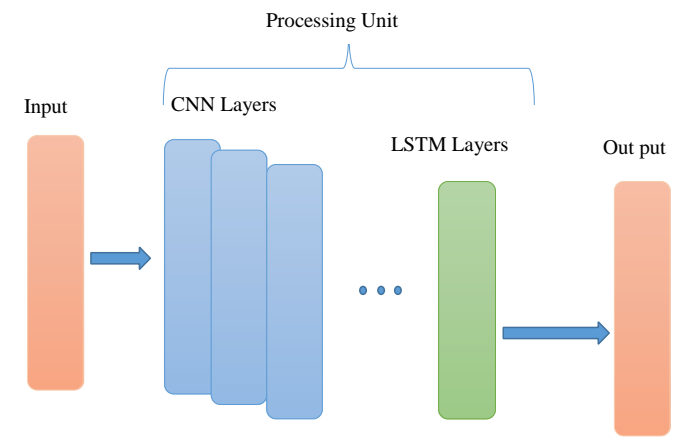

(b)

Figure 3. (a) Structure of CNN algorithm, and (b) structure of CNN-LSTM

\section{RESULTS AND DISCUSSION}

In this section, we discuss the results obtained from using the CNN and CNN-LSTM algorithms to recognize daily activities. As mentioned in section 2, six types of activities are analyzed by these algorithms i.e. "Walking", "Jogging", "Upstairs"," Downstairs", "Sitting" and "Standing". The analysis results are summarized in Figure 4, Figure 5 and Table 1. Figure 4 presents a comparison between the accuracy of these algorithms with respect to each activity. Accordingly, both of these algorithms are able to recognize activities with at least 90\% accuracy. This is important regarding the fact that WISDM is an imbalanced dataset. However, the CNN-LSTM algorithm has a higher level of accuracy. This superiority could be related to the fact that LSTM can consider timing dependencies among the input data.

Furthermore, the results presented in Figure 4 indicate that Jogging is the easiest activity to recognize by either of the algorithms, since it involves more changes in the acceleration parameter. On the other hand, recognition of going upstairs/downstairs is more difficult for these algorithms, since there is no input data about the direction of movement.

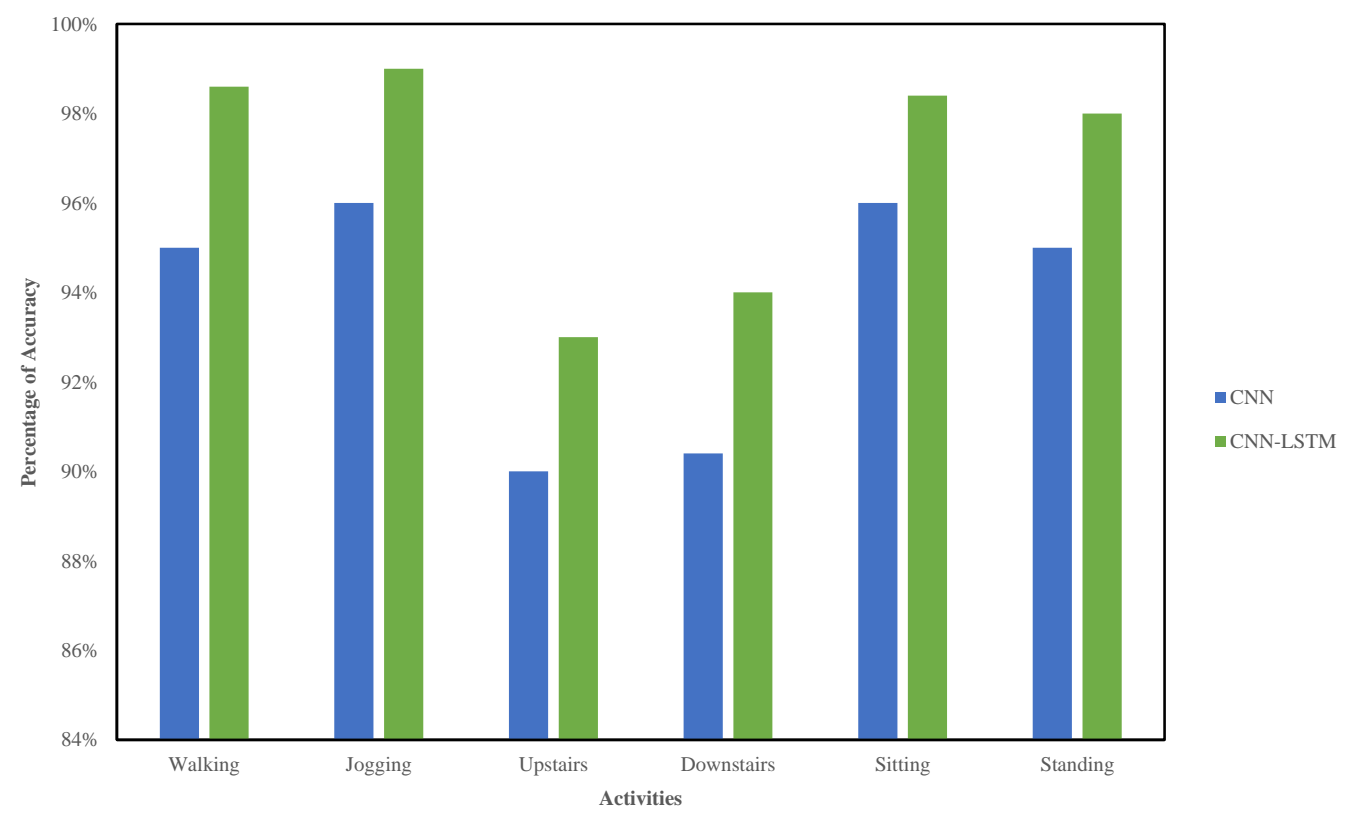

Figure 4. The accuracy of deep learning algorithms for different activities 
Table 1 compares the algorithms with respect to loss value, number of layers and epochs, and average accuracy. Both algorithms have the same number of convolution layers, and as expected, the CNN-LSTM algorithm has a higher accuracy and a less error ratio in average. Figure 5 indicates the relation between the number of epochs and the loss and accuracy of each algorithm. The CNN algorithm shows the optimal performance after 200 epochs of training. However, the CNN-LSTM algorithm needs 50 more epochs to reach the highest accuracy. Form the loss value viewpoint, we witness a steady decrease for both algorithms as we increase the number of epochs.

Table 1. The number of layers, Error and Accuracy values in different deep algorithms

\begin{tabular}{|c|c|c|c|c|}
\hline Type of algorithm & Epochs & Error & Layers & Average Accuracy \\
\hline$C N N$ & 200 & 0.15 & 4 & $94 \%$ \\
\hline CNN-LSTM & 250 & 0.12 & 5 & $97 \%$ \\
\hline
\end{tabular}

Achieving high accuracy in learning motion activities is very important, especially in the context of mHealth systems. For example, assume that we have some patients suffering from Parkinson's disease, and they are supposed to perform some rehabilitation activities to improve their bodily functions. These activities help patients get their abilities back and regain independence. If we have a mHealth system capable of accurately recognizing these activities and giving the patients feedback in case of some mistakes, then they would be able to improve their health with less need to go to clinics.

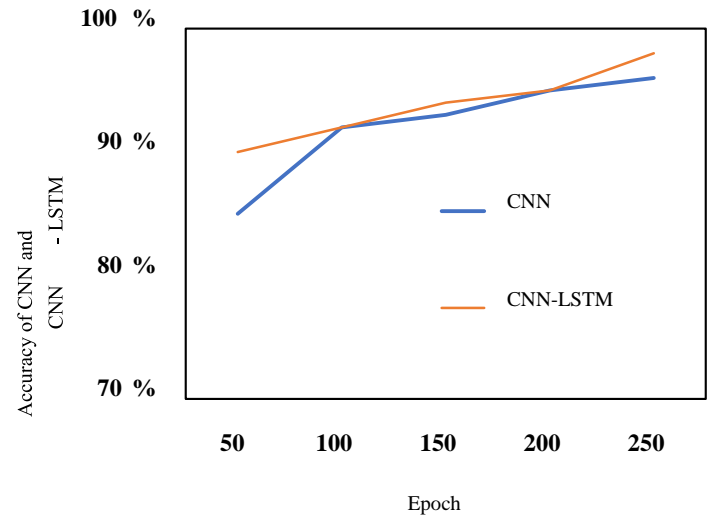

(a)

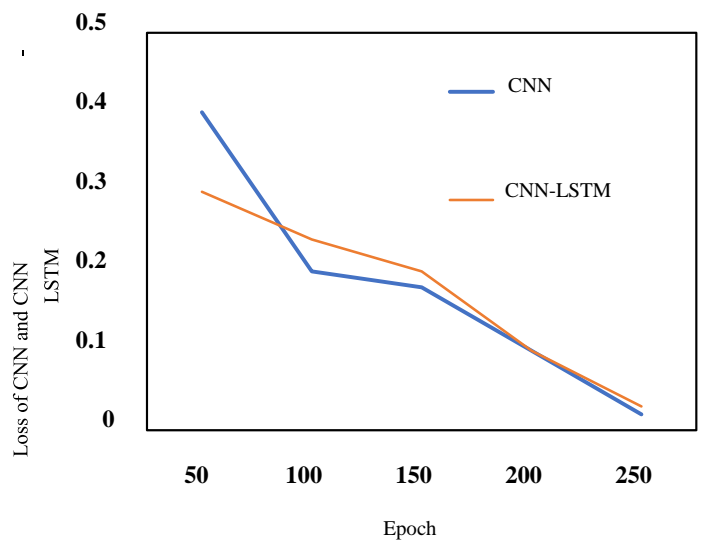

(b)

Figure 5. (a) The accuracy of deep learning algorithms, and (b) The loss of CNN and CNN-LSTM

Despite the advantages mentioned above, our proposed research has several limitations. First, the WISDM dataset is a small dataset and includes a few types of activities. Second, the data stored in the WISDM dataset is collected by a smartphone. In future work, we are going to use wearable sensors to collect more useful data and analyze the accuracy of deep learning algorithms. Third, we focused on only two deep learning algorithms i.e. CNN and CNN-LSTM in this research. Despite the strength and wide application of these models, we plan to examine other deep learning algorithms e.g. Gated Recurrent Units (GRUs) in our experiments. 


\section{CONCLUSION}

In this paper, we presented the results of an experiment on the application of deep learning algorithm in the context of human activity recognition. In this experiment, the data recorded in the WISDM dataset were used for training two deep learning models. The first model included four layers of CNNs, but the second model was a combination of four CNN layers and one LSTM layer. The experiment results indicated that both of the models were able to recognize daily activities with a high level of accuracy i.e. more than $94 \%$. However, the second algorithm performed more accurately.

\section{REFERENCES}

Adibi, S. (Ed.). (2015). Mobile health: a technology road map (Vol. 5). Springer.

Altini, M., Penders, J., Vullers, R., \& Amft, O. (2014). Estimating energy expenditure using body-worn accelerometers: a comparison of methods, sensors number and positioning. IEEE journal of biomedical and health informatics, 19(1), 219-226.

Asimina, S., Chapizanis, D., Karakitsios, S., Kontoroupis, P., Asimakopoulos, D. N., Maggos, T., \& Sarigiannis, D. (2018). Assessing and enhancing the utility of low-cost activity and location sensors for exposure studies. Environmental monitoring and assessment, 190(3), 155.

Boletsis, C., McCallum, S., \& Landmark, B. F. (2015, August). The use of smartwatches for health monitoring in home-based dementia care. In International Conference on Human Aspects of IT for the Aged Population (pp. 15-26). Springer, Cham.

Carneiro, S., Silva, J., Aguiar, B., Rocha, T., Sousa, I., Montanha, T., \& Ribeiro, J. (2015, May). Accelerometer-based methods for energy expenditure using the smartphone. In 2015 IEEE International Symposium on Medical Measurements and Applications (MeMeA) Proceedings (pp. 151-156). IEEE.

Duclos, M., Fleury, G., Lacomme, P., Phan, R., Ren, L., \& Rousset, S. (2016). An acceleration vector variance based method for energy expenditure estimation in real-life environment with a smartphone/smartwatch integration. Expert Systems with Applications, 63, 435-449.

Fan, X., Huang, H., Qi, S., Luo, X., Zeng, J., Xie, Q., \& Xie, C. (2015). Sensing home: a cost-effective design for smart home via heterogeneous wireless networks. Sensors, 15(12), 30270-30292.

Fotouhi-Ghazvini, F., \& Abbaspour, S. (2020). Wearable wireless sensors for measuring calorie consumption. Journal of Medical Signals and Sensors, 10(1), 19.

Gusenbauer, D., Isert, C., \& Krösche, J. (2010, September). Self-contained indoor positioning on off-the-shelf mobile devices. In 2010 International Conference on Indoor Positioning and Indoor Navigation (pp. 1-9). IEEE.

Hamari, L., Kullberg, T., Ruohonen, J., Heinonen, O. J., Díaz-Rodríguez, N., Lilius, J., ... \& Salanterä, S. (2017). Physical activity among children: Objective measurements using Fitbit One ${ }^{\circledR}$ and ActiGraph. BMC research notes, 10(1), 161.

Hwang, P. Y., Chou, C. C., Fang, W. C., \& Hwang, C. M. (2016, May). Smart shoes design with embedded monitoring electronics system for healthcare and fitness applications. In 2016 IEEE International Conference on Consumer Electronics-Taiwan (ICCE-TW) (pp. 1-2). IEEE.

Jain, Y., Chowdhury, D., \& Chattopadhyay, M. (2017, December). Machine learning based fitness tracker platform using mems accelerometer. In 2017 International Conference on Computer, Electrical \& Communication Engineering (ICCECE) (pp. 1-5). IEEE.

King, C. E., \& Sarrafzadeh, M. (2018). A survey of smartwatches in remote health monitoring. Journal of healthcare informatics research, 2(1-2), 1-24.

Kwapisz, J. R., Weiss, G. M., \& Moore, S. A. (2011). Activity recognition using cell phone accelerometers. ACM SigKDD Explorations Newsletter, 12(2), 74-82.

Lee, H., Kim, E., Lee, Y., Kim, H., Lee, J., Kim, M., ... \& Yoo, S. (2018). Toward all-day wearable health monitoring: An ultralow-power, reflective organic pulse oximetry sensing patch. Science advances, 4(11), eaas9530.

Li, M., Kwak, K. C., \& Kim, Y. T. (2016). Estimation of energy expenditure using a patch-type sensor module with an incremental radial basis function neural network. Sensors, 16(10), 1566.

Pande, A., Zhu, J., Das, A. K., Zeng, Y., Mohapatra, P., \& Han, J. J. (2015). Using smartphone sensors for improving energy expenditure estimation. IEEE journal of translational engineering in health and medicine, 3, 1-12.

Schneider, M., \& Chau, L. (2016). Validation of the Fitbit Zip for monitoring physical activity among free-living adolescents. BMC research notes, 9(1), 448. 
Sukaphat, S. (2013). An applying of accelerometer in Android platform for controlling weight. In Diambil dari: http://ibacconference. org (No. 20\&, p. 20).

Van Camp, C. M., \& Hayes, L. B. (2017). Identifying beneficial physical activity during school recess: Utility and feasibility of the Fitbit. Journal of Behavioral Education, 26(4), 394-409.

Vathsangam, H., \& Sukhatme, G. S. (2015). U.S. Patent No. 8,965,402. Washington, DC: U.S. Patent and Trademark Office. 8,965,402.

Rundle, A. G., Sheehan, D. M., Quinn, J. W., Bartley, K., Eisenhower, D., Bader, M. M., ... \& Neckerman, K. M. (2016). Using GPS data to study neighborhood walkability and physical activity. American journal of preventive medicine, 50(3), e65-e72.

Choi, E. J., \& Kang, S. W. (2018). The relationship between acceptance intention toward a smartphone healthcare application and health-promoting behaviors among nursing students. CIN: Computers, Informatics, Nursing, 36(10), 494-500. 